\title{
The Effect of Extracorporeal Shockwave Therapy on de Quervain Tenosynovitis; a Clinical Trial
}

\author{
Shila Haghighat (iD) ${ }^{1}$, Babak Vahdatpour (iD ${ }^{1,{ }^{*}}$ and Elham Ataei (iD) ${ }^{1}$ \\ ${ }^{1}$ Department of Physical Medicine and Rehabilitation, School of Medicine, Isfahan University of Medical Sciences, Isfahan, Iran \\ "Corresponding author: Department of Physical Medicine and Rehabilitation, School of Medicine, Isfahan University of Medical Sciences, Isfahan, Iran. Email: \\ vahdatpour@med.mui.ac.ir
}

Received 2020 June 29; Revised 2020 October 10; Accepted 2020 October 13.

\begin{abstract}
Background: De Quervain tenosynovitis is mainly managed by conservative treatments, such as anti-inflammatory drugs, splint, and injection; however, surgical treatments are also recommended.

Objectives: The present study aimed at assessing the efficacy of extracorporeal shockwave therapy in the treatment of de Quervain tenosynovitis.

Methods: The current clinical trial was conducted on 26 patients with de Quervain tenosynovitis eligible for treatment. The intervention group received extracorporeal shock wave therapy (1000 impulses, $2 \mathrm{bar}, 15 \mathrm{~Hz}$ ), and the sham group a treatment without shock wave. Both groups received the same conservative treatments as thumb spica splint and $200 \mathrm{mg}$ celecoxib once daily for three weeks. The assessment instruments included the disabilities of the arm, shoulder, and hand (DASH) questionnaire, the visual analog scale (VAS), and the hand-grip strength test performed before and after treatment.

Results: After treatment, the DASH and VAS scores decreased, while the hand-grip strength increased significantly in the groups. DASH and VAS scores were significantly lower in the intervention group than the sham group after treatment $(\mathrm{P}<0.05)$.

Conclusions: Extracorporeal shockwave therapy is a safe and easy method to reduce pain and enhance upper extremity functions and hand-grip strength in patients with de Quervain tenosynovitis.
\end{abstract}

Keywords: Pain, Disability, Grip Strength, de Quervain Tenosynovitis, Shockwave

\section{Background}

De Quervain tenosynovitis was first described in 1985 by Fritz de Quervain (1). The extensor retinaculum holds the tendons of the extensor muscles in place and covers the first dorsal compartment, a fibro-osseous tunnel. Chronic overuse of the wrist may lead to swelling and thickening of the ligaments of the extensor retinaculum. As a result, entrapment of tendons, which pass through the first dorsal compartment of the wrist (under the fibers of the extensor retinaculum and over the radial styloid process), causes pain. Abductor pollicis longus and the extensor pollicis brevis tendons are affected by this condition (2-4). Pain and swelling on the thumb side of the wrist, impaired function of the thumb, and gripping problems are the most common symptoms of the de Quervain disease, worsening with an increase in the motion of the thumb $(5,6)$. Its prevalence, at the highest rate, is estimated $0.5 \%$ in males and $1.3 \%$ in females aged $40-60$ years $(7,8)$.

Diagnosis is based on clinical manifestations and examinations, such as the Finklestein test, revealing the pos- sibility of restricted tendon movement through a mixed maneuver, including ulnar deviation and first metacarpophalangeal joint flexion $(3,4)$. Other diagnostic tests, such as radiography, might be used to differentiate it from other causes of wrist pain, such as osteoarthritis and fractures (9).

Different therapies are suggested to treat de Quervain tenosynovitis; nonsteroidal anti-inflammatory drugs, injection of corticosteroids, immobilization with a splint, iontophoresis, therapeutic pulsed ultrasound, activity modification, and surgery are the main treatments of choice, which their outcomes are discussed in various studies $(6,10,11)$. Along with these well-known therapies for musculoskeletal disorders, new treatment methods are suggested and evaluated every day, one of which is extracorporeal shockwave therapy. Primary studies on the efficacy of this modality in fracture healing utilized animal models (12-14).

Although the mechanism of action of extracorporeal shockwave therapy is not yet fully understood (15), satis- 
fying outcomes made it a treatment of choice for various orthopedic disorders, such as plantar fasciitis, bone fractures, osteonecrosis, and tendinopathies (16-19). Although a few studies suggested that shockwave therapy is not as effective as non-invasive techniques, it is a satisfying, safe, and effective procedure with low complications compared to its advantages $(15,20-23)$. Studies evaluating the effect of shockwave therapy on different types of tenosynovitis are limited and, to the best of authors' knowledge, only one study discussed its effects on de Quervain tenosynovitis thus far (24).

\section{Objectives}

Therefore, the present study aimed at assessing the efficacy of extracorporeal shockwave therapy in the treatment of de Quervain tenosynovitis.

\section{Methods}

\subsection{Study Design}

The current controlled, randomized, clinical trial was conducted on 26 patients ( $n=13$ in each group) with de Quervain tenosynovitis referred to physical medicine and rehabilitation centers affiliated to Isfahan University of Medical Sciences from 2015 to 2018.

\subsection{Inclusion and Exclusion Criteria}

The inclusion criteria were: receiving a diagnosis of de Quervain tenosynovitis, age over 18 years, and no history of undergoing physiotherapy modalities or corticosterone injection therapies for any reasons during the past month. All the patients completed the written informed consent form for participation in the study.

De Quervain tenosynovitis was diagnosed based on the standard criteria, including pain and tenderness in the lateral wrist and a positive result for the Finkelstein test. However, subjects were excluded from the study in case of other neurological disorders and rheumatologic diseases causing wrist pain, pregnancy, history of fracture or surgery on the hand, severe trauma, and coagulation disorders. Also, patients unwilling to continue the study during treatment, the ones with severe or acute complications after the intervention, and the ones who needed to alter the treatment were excluded from the study. At the study onset, demographic information of patients, including age, gender, and duration of disease, was recorded. Afterward, the patients were randomly divided into two parallel groups as extracorporeal shock wave therapy and sham by Random Allocation Software through the blocking method. The study had a single-blinded design, and patients were blind to allocations (Figure 1).

\subsection{Clinical Assessments}

In the shock wave therapy group, each patient first sat on a chair, and the hand with the disorder was placed on a surface. After rubbing the gel, a conductive medium to guide the waves, the probe of the device (Duolith ${ }^{\circledR}$ SD1, STORZ, Switzerland) was placed on the spot to transmit the waves to the site. There were three therapeutic sessions with a week interval for each patient. The treatment protocol utilized in the intervention group was 1000 impulses with 2 bar pressure at a frequency of $15 \mathrm{~Hz}$ on the radial head and focused on the tissue surrounding the maximal pain point. The treatment regimens in the sham group were similar to those of the intervention group with a turned-off device without induction of shock waves (sham shock wave). In both the intervention and sham groups, conservative treatments included administration of an immobilized splint of the thumb and an effective dose of an anti-inflammatory agent, such as celecoxib (200 mg daily), for two weeks.

Before and after the treatment, the disabilities of the arm, shoulder, and hand (DASH) questionnaire, the visual analogue scale (VAS), and the hand-grip strength test were used to evaluate the patients in both the intervention and sham groups. DASH scale includes 30 items on the symptoms and function of the upper extremity involved with orthopedic and neurological disorders, scored based on a five-point Likert scale, ranging from 1 (no stiffness or no symptoms) to 5 (an inability to perform activities or the most severe symptoms). The total score ranges from 30 to 150 (25).

The VAS, scored from 0 to 10 , was used to evaluate the intensity of pain in the wrist, and the hand-grip strength test was performed using a hand-grip dynamometer in all patients. The procedures were explained and demonstrated to the patients. The patient was seated comfortably on a chair with a forearm resting on the table. Then they were asked to hold the dynamometer and pull the handle, and accordingly, the highest voluntary contraction was recorded (26). The patients were followed up for two months and visited on arrival and immediately after the completion of treatment (after three weeks), as well as three and six weeks after the treatment cessation, and the patient outcomes were evaluated in these visits using the assessment tools.

The research proposal of the present study was confirmed (ID no.: 395805), and its protocol was approved by the Ethics Committee of Isfahan University of Medical Sciences (ethical code: IR.mui.rec.1395.3.805).

\subsection{Statistical Analysis}

The sample size was calculated based on a confidence interval of $90 \%$, a test power of $75 \%$, a standard deviation 


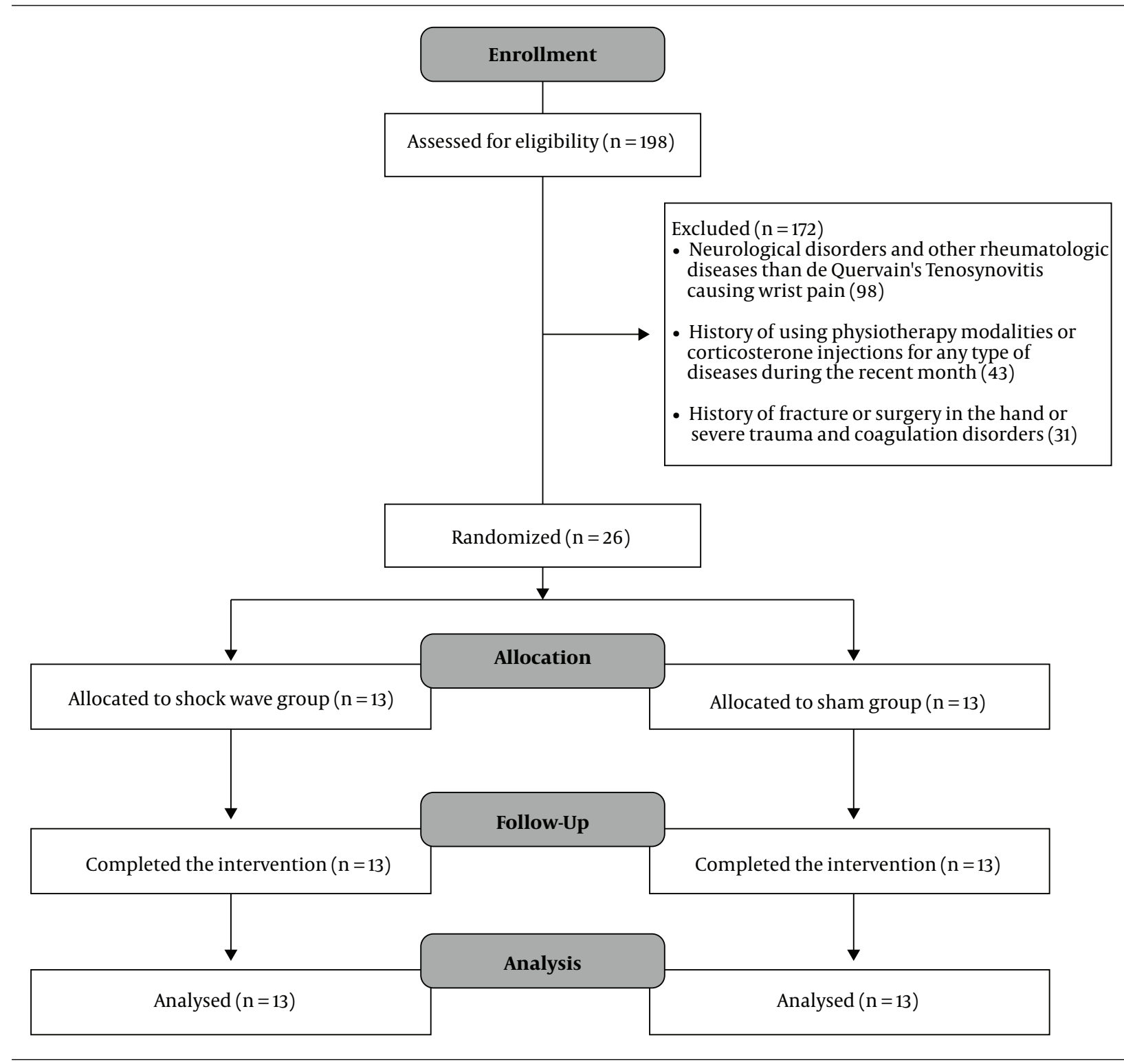

Figure 1. Consort flow diagram of the study

of 0.9, and mean differences of 0.3. The IBM SPSS software for Windows (SPSS Inc., Chicago, IL, USA, version 20) was used to analyze the data. The data were expressed as mean \pm standard deviation (SD) or frequency (percentage). The Shapiro-Wilk test was used to assess the normality of data distribution. The results showed that the residuals of hand-grip strength (in all four times) and VAS (before treatment) did not have a normal distribution $(\mathrm{P}<0.05)$. However, the residuals of DASH (in all four times) and VAS (after the completion of treatment, as well as three and six weeks after the cessation of treatment) had a normal distribution according to the results of the Shapiro-Wilk test ( $\mathrm{P}>$
0.05). The independent samples $t$-test was also used for intragroup comparisons, and according to the results, their residuals had a normal distribution. The Mann-Whitney test was also performed for data with non-normal distribution residuals. Moreover, the repeated-measures ANOVA was used to compare pre-and post-VAS and DASH measures and the Friedman test to compare hand-grip strength data. Also, the chi-Squared test was employed for intergroup comparison of qualitative data. Changes in the variables were compared by the repeated measures ANOVA. A P-value of $<0.05$ was considered as the level of significance. 


\section{Results}

In the current study, 26 patients were divided into the intervention (six males and seven females with a mean age of $44.61 \pm 11.36$ years) and sham (four males and nine females with a mean age of $48.23 \pm 14.45$ years) groups. There were no significant differences between the groups in terms of age, gender, involved side, and history of the disease $(\mathrm{P}>0.05)$. All the patients completed the study (Table 1). Changes in VAS scores were significant at different intervals. The VAS score in the intervention group significantly reduced after the intervention $(\mathrm{P}<0.05)$, but the changes were insignificant in the sham group $(\mathrm{P}>0.05)$. The means of VAS score were significantly lower in the intervention group after treatment as well as three and six weeks after treatment cessation than those of the sham group $(\mathrm{P}<0.05)$. However, there was no significant difference between the groups in terms of VAS score before treatment $(\mathrm{P}>0.05)$. DASH score significantly decreased in both groups six weeks after treatment cessation, and the means of DASH score were significantly lower in the intervention group than sham after treatment completion as well as three and six weeks after the cessation of treatment $(\mathrm{P}<0.05)$. However, the difference was insignificant before the treatment onset $(\mathrm{P}>0.05)$. The hand-grip strength in both the intervention and sham groups increased significantly six weeks after the treatment cessation $(\mathrm{P}<0.05)$; however, the difference was insignificant at all intervals ( $P$ $>0.05$ ) (Tables 2 and 3).

\begin{tabular}{lccc}
\hline \multicolumn{4}{l}{ Table 1. Demographic Data of Patients in Both Groups } \\
\hline Demographics & Intervention $(\mathbf{N}=\mathbf{1 3})$ & Sham $(\mathbf{N}=\mathbf{1 3})$ & P-Value \\
\hline Age, $\mathbf{y}$ & $44.61 \pm 11.36$ & $48.23 \pm 14.45$ & 0.43 \\
Gender (M/F) & $6 / 7$ & $4 / 9$ & 0.42 \\
Involved side (R/L) & $7 / 6$ & $5 / 8$ & 0.43 \\
History, $\mathbf{y}$ & $25.69 \pm 8.46$ & $20.84 \pm 4.87$ & 0.43 \\
\hline
\end{tabular}

${ }^{\mathrm{a}}$ Values are expressed as mean $\pm \mathrm{SD}$.

\section{Discussion}

The present study aimed at evaluating the effects of shockwave therapy on de Quervain tenosynovitis, and according to the obtained results, VAS score significantly decreased in the intervention group after the treatment completion, as well as three and six weeks after the treatment cessation, whereas no improvement was observed in the sham group at different intervals. These findings revealed that pain may persist with conservative treatment, and extracorporeal shockwave therapy could reduce it in patients with de Quervain tenosynovitis. Although some models are proposed for the mechanism of pain relief induced by shockwave therapy, the information about this subject is not very detailed (27-30). Extracorporeal shockwave therapy is applied to many musculoskeletal disorders since its benefits are proved based on the results of studies (31). Large areas and deep tissue can be targeted by the shockwave, and it does not depend on the reports of any imaging-based guidance system to determine the target area, which makes its utilization easier (31-34). Since it does not stimulate pain in the target area, and its effects might be blocked by local anesthetics, it is applied without local anesthetics $(31,35,36)$. It is believed that chronic pain caused by many conditions, such as de Quervin disease, is due to synaptic threshold modification creating memory reflex, and shockwave therapy can remove these memories (27). Therefore, pain relief may happen as the advantage of extracorporeal shockwave therapy, as demonstrated in the present study. DASH scores significantly reduced after the completion of treatment in both groups, but the differences were more significant in the intervention group. The obtained results demonstrated that conservative treatment could effectively improve the function of the upper limb in de Quervain tenosynovitis over time, but adding extracorporeal shockwave treatment significantly improved the outcomes since the scores significantly increased compared to the sham group just after the treatment completion and in follow-ups. In the present study, the results of the hand-grip strength test revealed a significant improvement after the completion of treatment with shockwave therapy. Likewise, conservative treatment was effective, and there were no significant differences between the groups, although shockwave therapy resulted in better outcomes. These results mean that although extracorporeal shockwave therapy improved hand-grip strength, almost the same results were obtained by conservative therapy, and deciding on the treatment of choice should rely on patient satisfaction with treatment and the costbenefit ratio. Elerain (24) conducted a similar study to compare the outcomes of radial shockwave therapy and physiotherapy on 32 patients with de Quervain tenosynovitis assigned to two groups. One group received an ultrasound at a frequency of $1 \mathrm{~Hz}$ and the intensity of 1.2 $\mathrm{W} / \mathrm{cm}^{2}$, and the other group underwent radial shockwave therapy at a frequency of $5 \mathrm{~Hz}$ and pressure of 1/5 MPa (15 bar). Findings of his study indicated that general pain decreased and pain during thumb and wrist movement reduced in both the groups. Also, duration and periodicity of pain decreased in both groups. These findings were not significantly different between the groups; however, the author suggested that shockwave therapy might lead to faster pain relief and complementary splinting, a key el- 
Haghighat S et al.

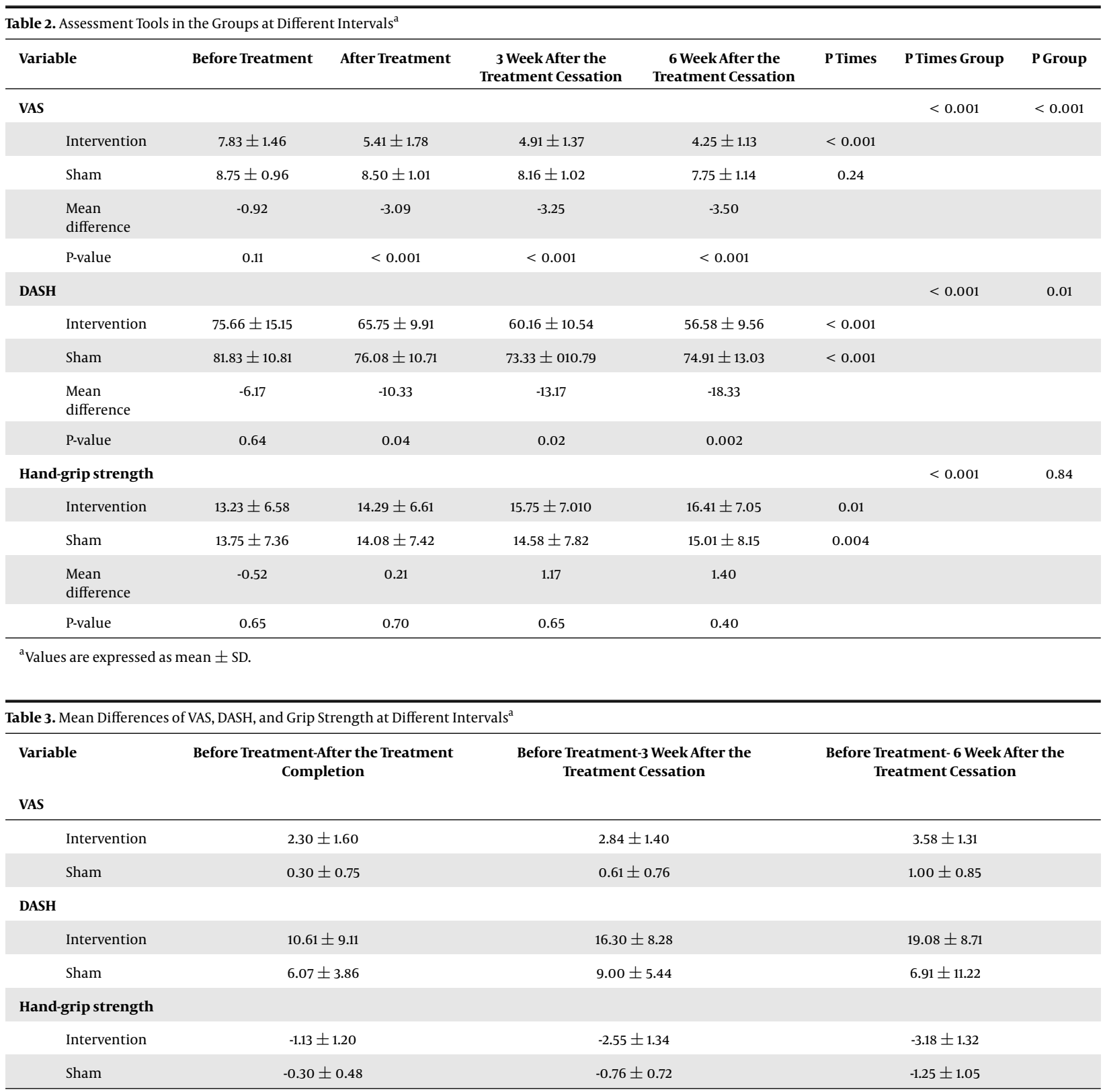

${ }^{\mathrm{a}}$ Values are expressed as mean $\pm \mathrm{SD}$.

ement in the efficacy of this type of treatment. Some of the differences between the study by Elerain (24) and the present one were the frequency, impulses, and intensity used for the shockwave group. Another difference was that three therapeutic sessions were used in the present study with a week interval for follow-up in both groups, whereas 10 sessions were held in his research for the physiotherapy group (two sessions per week) and three sessions for the shockwave group (without intervals). The control groups were also different in two studies; the present research im- mobilized the thumb with a splint and administered celecoxib whereas, in the study by Elerian (24), subjects underwent ultrasound therapy and active exercises for hand muscles. These differences might lead to different results, although it is agreed that shockwave therapy is efficient, especially in a shorter time. Similar results are reported in the studies using shockwave therapy to treat tenosynovitis and tendinopathies (37-39). The scarcity of complications, safety, effective pain relief, and rapid outcomes are the most notable benefits of this method, which were in 
line with the findings of the present study.

\subsection{Limitations}

The main limitation of the study was the small sample size due to the low prevalence of the disease between males and females. It is recommended to perform studies with larger sample sizes to compare different treatment methods for de Quervain tenosynovitis and evaluate multidimensional aspects of this disorder in terms of pain, upper limb function, and follow-up outcomes. Second, the effects of arm splint and anti-inflammatory drugs were not compared directly and separately from the shockwave therapy as the conservative treatment applied to both groups. Third, general pain was evaluated through VAS, and other detailed aspects of pain, such as duration and pain caused by different movements of the wrist, were not investigated. Fourth, the outcomes of other treatments, such as corticosteroid injection, physiotherapy, and surgical interventions, were not compared with those of shockwave therapy in the present study, although controlled clinical trials compared their outcomes with those of sham groups (10, 14, 40-42).

\subsection{Conclusions}

Extracorporeal shockwave therapy is a safe and easy method to reduce pain, enhance upper extremity functions, and strengthen hand-grip in patients with de Quervain tenosynovitis if accompanied by conservative therapies, such as thumb splint, and the outcomes may be achieved in a shorter time than other treatments used alone. Pain relief is more significantly achieved in this technique, as it may not be effectively observed in other methods alone. However, due to the small sample size, conclusive results cannot be obtained.

\section{Footnotes}

Authors' Contribution: Study concept and design: $\mathrm{SH}$. Analysis and interpretation of data: BV. Development of the protocol, abstraction of data, and writing of the manuscript: EA.

Clinical Trial Registration Code: The clinical trial registration code was IRCT20120729010439N2.

Conflict of Interests: The authors declared no conflicts of interest.

Ethical Approval: The ethical approval code was IR.mui.rec.1395.3.805.

Funding/Support: There was no financial support for research or manuscript production.

Informed Consent: All the patients signed the written informed consent form for participation in the study.

\section{References}

1. Aurélien Michel T, Pauline D, Debora S, Clémence B, Camillo Théo M, Swenn Maxence K. De Quervain Tenosynovitis in a 12-Year-Old Child: A Generation's Disease? J Orthop Surg Tech. 2017;1(2). doi: 10.36959/453/517.

2. Wolf JM, Sturdivant RX, Owens BD. Incidence of de Quervain's tenosynovitis in a young, active population. J Hand Surg Am. 2009;34(1):112-5. doi: 10.1016/j.jhsa.2008.08.020. [PubMed: 19081683].

3. Goel R, Abzug JM. De Quervain's tenosynovitis: a review of the rehabilitative options. Hand. 2015;10(1):1-5. doi: 10.1007/s11552-014-9649-3. [PubMed: 25762881].

4. Ilyas AM, Ast M, Schaffer AA, Thoder J. De quervain tenosynovitis of the wrist.J Am Acad Orthop Surg. 2007;15(12):757-64. doi:10.5435/00124635200712000-00009. [PubMed: 18063716].

5. Moore JS. De Quervain's tenosynovitis. Stenosing tenosynovitis of the first dorsal compartment. J Occup Environ Med. 1997;39(10):990-1002. doi:10.1097/00043764-199710000-00011. [PubMed: 9343764].

6. Patel KR, Tadisina KK, Gonzalez MH. De Quervain's disease. Eplasty. 2013;13.

7. Walker-Bone K, Palmer KT, Reading I, Coggon D, Cooper C. Prevalence and impact of musculoskeletal disorders of the upper limb in the general population. Arthritis Rheum. 2004;51(4):642-51. doi: 10.1002/art.20535. [PubMed: 15334439].

8. Hadianfard M, Ashraf A, Fakheri M, Nasiri A. Efficacy of acupuncture versus local methylprednisolone acetate injection in De Quervain's tenosynovitis: a randomized controlled trial. J Acupunct Meridian Stud. 2014;7(3):115-21. doi: 10.1016/j.jams.2013.10.003. [PubMed: 24929455].

9. Li YK, White CP. Five things to know about...carpometacarpal osteoarthritis of the thumb. CMAJ. 2013;185(2):149. doi: 10.1503/cmaj.111444. [PubMed: 23008487]. [PubMed Central: PMC3563889].

10. Huisstede BM, Coert JH, Friden J, Hoogvliet P, European HG. Consensus on a multidisciplinary treatment guideline for de Quervain disease: results from the European HANDGUIDE study. Phys Ther 2014;94(8):1095-110. doi:10.2522/ptj.20130069. [PubMed: 24700135].

11. Oosting K, Krenselewski B, Dolislager C. Effective Conservative Treatments for de Quervain's Tenosynovitis: A Retrospective Outcome Study. Grand Valley State University; 2013.

12. Haupt G, Haupt A, Ekkernkamp A, Gerety B, Chvapil M. Influence of shock waves on fracture healing. Urology. 1992;39(6):529-32. doi: 10.1016/0090-4295(92)90009-1. [PubMed: 1615601].

13. Johannes EJ, Kaulesar Sukul DM, Matura E. High-energy shock waves for the treatment of nonunions: an experiment on dogs. J Surg Res. 1994;57(2):246-52. doi: 10.1006/jsre.1994.1139. [PubMed: 8028332].

14. Oh JK, Messing S, Hyrien O, Hammert WC. Effectiveness of Corticosteroid Injections for Treatment of de Quervain's Tenosynovitis. Hand (N Y). 2017;12(4):357-61. doi: 10.1177/1558944716681976. [PubMed: 28644946]. [PubMed Central: PMC5484456].

15. Wang CJ. Extracorporeal shockwave therapy in musculoskeletal disorders.J Orthop Surg Res. 2012;7:11. doi: 10.1186/1749-799X-7-11. [PubMed: 22433113]. [PubMed Central: PMC3342893].

16. Wilner JM, Strash WW. Extracorporeal shockwave therapy for plantar fasciitis and other musculoskeletal conditions utilizing the Ossatron-an update. Clin Podiatr Med Surg. 2004;21(3):441-7. viii. doi: 10.1016/j.cpm.2004.03.002. [PubMed: 15246149].

17. Cacchio A, Giordano L, Colafarina O, Rompe JD, Tavernese E, Ioppolo F, et al. Extracorporeal shock-wave therapy compared with surgery for hypertrophic long-bone nonunions. J Bone Joint Surg Am. 2009;91(11):2589-97. doi: 10.2106/JBJS.H.00841. [PubMed:19884432]. 
18. Alves EM, Angrisani AT, Santiago MB. The use of extracorporeal shock waves in the treatment of osteonecrosis of the femoral head: a systematic review. Clin Rheumatol. 2009;28(11):1247-51. doi: 10.1007/s10067-009-1231-y. [PubMed: 19609482].

19. van Leeuwen MT, Zwerver J, van den Akker-Scheek I. Extracorporeal shockwave therapy for patellar tendinopathy: a review of the literature. Br J Sports Med. 2009;43(3):163-8. doi: 10.1136/bjsm.2008.050740. [PubMed: 18718975].

20. Buchbinder R, Green SE, Youd JM, Assendelft WJ, Barnsley L, Smidt N. Shock wave therapy for lateral elbow pain. Cochrane Database Syst Rev. 2005;(4). CD003524. doi:10.1002/14651858.CD003524.pub2. [PubMed: 16235324].

21. Ogden JA, Alvarez RG, Levitt R, Marlow M. Shock wave therapy (Orthotripsy) in musculoskeletal disorders. Clin Orthop Relat Res. 2001;(387):22-40. doi: 10.1097/00003086-200106000-00005. [PubMed: 11400888].

22. Vahdatpour B, Taheri P, Zade AZ, Moradian S. Efficacy of extracorporeal shockwave therapy in frozen shoulder. Int $J$ Prev Med. 2014;5(7):875-81. [PubMed: 25104999]. [PubMed Central: PMC4124565].

23. Moayednia A, Haghdani S, Khosrawi S, Yousefi E, Vahdatpour B. Longterm effect of extracorporeal shock wave therapy on the treatment of chronic pelvic pain syndrome due to non bacterial prostatitis. $J$ Res Med Sci. 2014;19(4):293-6. [PubMed: 25097599]. [PubMed Central: PMC4115342].

24. Elerian A. Effect of shockwaves versus traditional physiotherapy in treating de quervain tenosynovitis. 2016.

25. De Smet L. The DASH questionnaire and score in the evaluation of hand and wrist disorders. Acta Orthop Belg. 2008;74(5):575-81. [PubMed: 19058688].

26. Lusardi MM, Bohannon RW. Hand grip strength: Comparability of measurements obtained with a jamar dynamometer and a modified sphygmomanometer. J Hand Ther. 1991;4(3):117-22. doi: 10.1016/s08941130(12)80226-2.

27. Wess OJ. A neural model for chronic pain and pain relief by extracorporeal shock wave treatment. Urol Res. 2008;36(6):327-34. doi: 10.1007/s00240-008-0156-2. [PubMed: 18839163].

28. Schmitz C, DePace R. Pain relief by extracorporeal shockwave therapy: an update on the current understanding. Urol Res. 2009;37(4):231-4. doi: 10.1007/s00240-009-0190-8. [PubMed: 19444437]. [PubMed Central: PMC2714448].

29. Han H, Lee D, Lee S, Jeon C, Kim T. The effects of extracorporeal shock wave therapy on pain, disability, and depression of chronic low back pain patients. J Phys Ther Sci. 2015;27(2):397-9. doi: 10.1589/jpts.27.397. [PubMed: 25729177]. [PubMed Central: PMC4339147].

30. Lee S, Lee D, Park J. Effects of extracorporeal shockwave therapy on patients with chronic low back pain and their dynamic balance ability. J Phys Ther Sci. 2014;26(1):7-10. doi: 10.1589/jpts.26.7. [PubMed: 24567665]. [PubMed Central: PMC3927045].

31. Schmitz C, Csaszar NB, Milz S, Schieker M, Maffulli N, Rompe JD, et al. Efficacy and safety of extracorporeal shock wave therapy for orthopedic conditions: a systematic review on studies listed in the PEDro database. Br Med Bull. 2015;116:115-38. doi: 10.1093/bmb/ldv047.
[PubMed: 26585999]. [PubMed Central: PMC4674007].

32. Gerdesmeyer L, Frey C, Vester J, Maier M, Weil LJ, Weil LS, et al. Radial extracorporeal shock wave therapy is safe and effective in the treatment of chronic recalcitrant plantar fasciitis: results of a confirmatory randomized placebo-controlled multicenter study. Am J Sports Med. 2008;36(11):2100-9. doi: 10.1177/0363546508324176. [PubMed: 18832341].

33. Gerdesmeyer L, Gollwitzer H, Diehl P, Wagner K. Radial extracorporeal shockwave therapy (rESWT) in orthopaedics. J Miner Stoffwechs. 2004;11(4):36-9.

34. Kolk A, Yang KG, Tamminga R, van der Hoeven H. Radial extracorporeal shock-wave therapy in patients with chronic rotator cuff tendinitis: a prospective randomised double-blind placebo-controlled multicentre trial. Bone Joint J. 2013;95-B(11):1521-6. doi: 10.1302/0301620X.95B11.31879. [PubMed: 24151273].

35. Rompe JD, Meurer A, Nafe B, Hofmann A, Gerdesmeyer L. Repetitive low-energy shock wave application without local anesthesia is more efficient than repetitive low-energy shock wave application with local anesthesia in the treatment of chronic plantar fasciitis. J Orthop Res. 2005;23(4):931-41. doi: 10.1016/j.orthres.2004.09.003. [PubMed: 16023010].

36. Lohrer H, Nauck T, Dorn-Lange NV, Scholl J, Vester JC. Comparison of radial versus focused extracorporeal shock waves in plantar fasciitis using functional measures. Foot Ankle Int. 2010;31(1):1-9. doi: 10.3113/FAI.2010.0001. [PubMed: 20067715].

37. Leeman JJ, Shaw KK, Mison MB, Perry JA, Carr A, Shultz R. Extracorporeal shockwave therapy and therapeutic exercise for supraspinatus and biceps tendinopathies in 29 dogs. Vet Rec. 2016;179(15):385. doi: 10.1136/vr.103487. [PubMed: 27444781].

38. Malliaropoulos N, Jury R, Pyne D, Padhiar N, Turner J, Korakakis V, et al. Radial extracorporeal shockwave therapy for the treatment of finger tenosynovitis (trigger digit). Open Access J Sports Med. 2016;7:14351. doi: 10.2147/OAJSM.S108126. [PubMed: 27843364]. [PubMed Central: PMC5098764].

39. Notarnicola A, Moretti B. The biological effects of extracorporeal shock wave therapy (eswt) on tendon tissue. Muscles Ligaments Tendons J. 2012;2(1):33-7. [PubMed: 23738271]. [PubMed Central: PMC3666498].

40. Huisstede BM, Gladdines S, Randsdorp MS, Koes BW. Effectiveness of Conservative, Surgical, and Postsurgical Interventions for Trigger Finger, Dupuytren Disease, and De Quervain Disease: A Systematic Review. Arch Phys Med Rehabil. 2018;99(8):1635-1649 e21. doi: 10.1016/j.apmr.2017.07.014. [PubMed: 28860097].

41. Rowland P, Phelan N, Gardiner S, Linton KN, Galvin R. The Effectiveness of Corticosteroid Injection for De Quervain's Stenosing Tenosynovitis (DQST): A Systematic Review and Meta-Analysis. Open Orthop J. 2015;9:437-44. doi: 10.2174/1874325001509010437. [PubMed: 26587059]. [PubMed Central: PMC4655850].

42. Makarawung DJ, Becker SJ, Bekkers S, Ring D. Disability and pain after cortisone versus placebo injection for trapeziometacarpal arthrosis and de Quervain syndrome. Hand (N Y). 2013;8(4):375-81. doi: 10.1007/s11552-013-9529-2. [PubMed: 24426952]. [PubMed Central: PMC3840768]. 